\title{
Design of a Three Dimensional Magnetic Field Measurement System for TMS Pre-Testing
}

\author{
Hui Xiong ${ }^{1 *}$, Jianguo Zhu ${ }^{2 *}$, Jinhua Shi ${ }^{1}$, Shuai Meng ${ }^{1}$, and Xiaohui Luo ${ }^{1}$ \\ ${ }^{1}$ Tianjin Polytechnic University, Tianjin, People's Republic of China \\ ${ }^{2}$ University of Technology Sydney, Australia \\ Email: fine_xh@163.com
}

\begin{abstract}
In the course of treatment and diagnosis of transcranial magnetic stimulation (TMS), the target and non-target are stimulated simultaneously, which produces undesirable side effects. To reduce the risk of such side effects, it is essential to test the stimulation before the real treatment. This paper presents the structure of a magnetic field measurement system for TMS pre-testing. The 3D magnetic field measurement structure can use a 3D magnetic sensor to measure the value of electromagnetic field in some specific points of the brain model to reduce the risk that could be caused by directly measuring in human brain and effectively avoid the side effects of TMS.
\end{abstract}

Index Terms-- Brain model, magnetic field measurement system, TMS pre-testing, 3D magnetic sensor

\section{INTRODUCTION}

The TMS is a painless, non-invasive, green treatment and diagnosis technique [1]. The principle of TMS is that a stimulating coil with pulse current is placed over the head. Based on Biot-Savart law, a time-varying current pulse can produce a magnetic field. The time-varying magnetic field, in turn, induces an electric field in the human head according to Faraday's law. The induced electric field can cause depolarization or hyperpolarization of the cells. And then a series of physiological and biochemical reactions will be followed [2], [3]. The magnetic stimulation is mainly used in the field of nerve medicine and rehabilitation medicine [4], [5]. Clinical study of TMS showed that the technology may make the subjects feel unwell in the process of treatment and diagnosis [6]-[8].

In order to avoid such side effects, it is desirable to check the field strength of stimulating spot before treatment. This paper presents the structure of a magnetic field measurement for TMS. The structure consists of three parts: a simplified brain model, three dimensional (3D) magnetic field measurement sensor (MFMS), and a 3D solenoid calibration-platform. The system uses a 3D array sensor to measure the magnetic field distribution of the simplified brain model in real time. According to the magnetic field distribution, the coil parameters (turns, shape and number) [9], [10], amplitude and magnitude of pulse current [11], [12] and the relative position of the coil and the stimulating target [13], [14] are adjusted in order to realize the optimal treatment effect. By this method, the pre-testing system effectively avoid the clinical risk.

\section{DESIGN}

\section{A. Simplified Brain Model}

A simplified brain model is one of the major parts of the $3 \mathrm{D}$ magnetic field measurement structure for TMS pre-testing [15]. The size of the adult skull model is about $19 \mathrm{~cm} \times 15 \mathrm{~cm} \times 21 \mathrm{~cm}$ and the shape of the brain is similar to an ellipsoid. So, the brain model can be simplified as an inscribed cuboid of ellipsoid with $14 \mathrm{~cm}$ $\times 9.5 \mathrm{~cm} \times 7 \mathrm{~cm}$ size.

Different brain regions have different dielectric parameters [16], so it is necessary for the simplified brain model to be divided into multiple small cuboid regions. Due to the limitation of the sensor size, the simplified head model is finally divided into 88 small $2 \mathrm{~cm} \times 2 \mathrm{~cm} \times$ $1.5 \mathrm{~cm}$ cuboids as shown in Fig. 1. A small cuboid structure has two functions. One is as a container injected different dielectric parameter solutions to replace different brain tissues, the other is placed a 3D MFMS as a container. The MFMS is isolated from the solution. When 88 3D MFMS are placed in 88 small cuboids, the 3D array MFMS structure is formed.

\section{B. The $3 D \mathrm{MFMS}$}

The 3D MFMS is composed of three mutual orthogonal square coils. The three coils have the same number of turns and the different areas of cross-sections.

In order to wind the sensor coils, the 3D MFMS frame is designed as shown in Fig. 2. The frame structure is obtained by digging a closed groove respectively around $\mathrm{X}, \mathrm{Y}$ and $\mathrm{Z}$ axis in the center of the cube. The edge length of the cube is $5 \mathrm{~mm}$ and the dimensions of the three closed grooves are listed in Table I. The closed grooves are used for winding the 3D coils of sensor. The winding directions of the three coils follow the right-hand rule. The coils will be wound by the sequence of $\mathrm{X}, \mathrm{Y}$ and $\mathrm{Z}$ axis. Thus, the cross-sectional areas of the $X, Y$, and $Z$ axis coils gradually increase.

The diameter of the enameled wire is $0.05 \mathrm{~mm}$. There is a difference in the turns of each coils during winding. The reason for this is that the size of the MSMF frame is too small, so the processing of coil winding may be limited. The coil in each closed grooves will been wound 2 layers, about 30 turns with one layer and about 60 turns in total.

\section{The 3D Solenoid Calibration-platform}

The $3 \mathrm{D}$ solenoid calibration-platform is designed for 


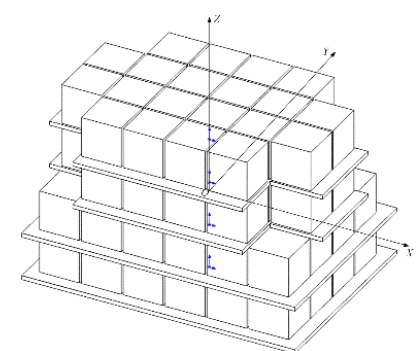

Fig. 1. Simplified divided brain model

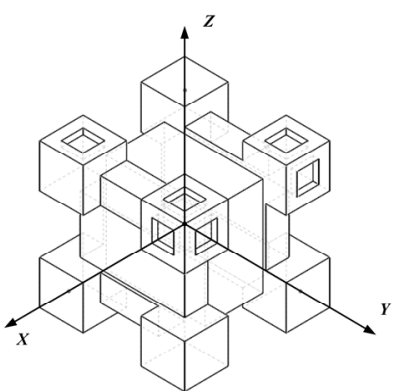

Fig. 2. The structure of the 3D MFMS frame (The number of the top small hollows represents the positive direction of the axis)

the calibration of sensor coefficient purpose. The platform consists of six identical and independent solenoids. As shown in Fig. 3, the single solenoid outer diameter is 50 $\mathrm{cm}$ and is wound 32 turns by enameled wire of $2 \mathrm{~cm}$ diameter.

The six solenoids are divided into three groups by $\mathrm{X}, \mathrm{Y}$ and $\mathrm{Z}$ axis. Each group of which is perpendicular to the two other groups, and the distance between the two solenoids in a group is $56 \mathrm{~cm}$. Three dimension array magnetic field measurement sensor system are placed in the central area of the calibration platform.

\section{Fundamental PRINCIPLE}

The stimulating coils with pulsed current are placed over the simplified brain model during TMS pre-testing experiment. In the simplified model, 3D MFMS can induced EMF. According to Faraday's law, the sensor coefficient $K_{c}$ which is the relationship of EMF $e(t)$ and the rate of magnetic flux density change $d B(t) / d t$ can be derived. The sensor coefficient $K_{c}$ is a constant, and $e(t)$ can be stored by data acquisition card. So, If the $K_{c}$ has been previously calibrated, $B(t)$ can be calculated.

To calibrate the coefficient constant $K_{c}$, the cosine current $i(t)$ is passed in the solenoids, the $e(t)$ of sensors can be measured and $B(t)$ of every sensor point needs to be obtained beforehand. In order to obtain the known $B(t)$, three dimension solenoid calibration-platform is needed to calibrate the solenoid coefficient $K_{s}$ that is the relationship of the solenoid current $I$ and magnetic flux density $B$ at the points of $3 \mathrm{D}$ sensors center.

\section{A. The Calibration Principle of 3D Solenoid Calibration-platform Coefficient}

The basic unit of solenoid can be regarded as circular coil as shown in Fig. 4. Based on the Biot-Savart law, the magnetic flux density at point $P$ satisfies the following equation:
TABLE I

The Dimensions of THE Closed GRoOves AROUND AXIS

\begin{tabular}{cccc}
\hline \hline Groove & $\begin{array}{c}\text { X-axis } \\
(\mathrm{mm})\end{array}$ & $\begin{array}{c}\text { Y-axis } \\
(\mathrm{mm})\end{array}$ & $\begin{array}{c}\text { Z-axis } \\
(\mathrm{mm})\end{array}$ \\
\hline Width & 1.5 & 1.5 & 1.5 \\
Depth & 0.8 & 0.6 & 0.4 \\
$\begin{array}{c}\text { Cross-sectional } \\
\text { area }\end{array}$ & $3.4 \times 3.4$ & $3.8 \times 3.8$ & $4.2 \times 4.2$ \\
\hline \hline
\end{tabular}

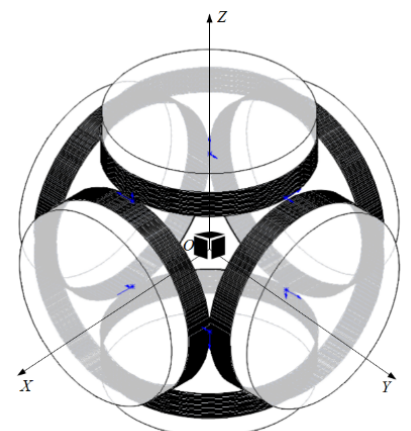

Fig. 3. Structure of 3D MFMS calibration platform

$$
\vec{B}=\int_{C} d \vec{B}=\frac{\mu_{0}}{4 \pi} \int_{C} \frac{I d \vec{l} \times \vec{R}}{R^{3}}
$$

where $\vec{B}=$ magnetic flux density vector, $C=$ integral path, $\mu_{0}=$ free-space permeability, $I=$ source current, $R=$ distance between point $P$ and current element $I d l$.

In the spherical ( $r, \theta, \beta$ components) coordinate system, the equation of point $P$ is

$$
P(x=r \sin \theta \cos \beta, y=r \sin \theta \sin \beta, z=r \cos \theta)
$$

The parametric $(\varphi)$ equation of the circular coil $C$ is convenient to use the expression

$$
(x=a \cos \varphi, y=a \sin \varphi, z=0)
$$

According to Eq. (1) - (3), the magnetic flux density B of a circular coil $C$ at point $P$ can be written as

$$
B=|\vec{B}|=\sqrt{B_{x}^{2}+B_{y}^{2}+B_{z}^{2}}
$$

where

$$
\begin{aligned}
& B_{x}=\frac{\mu_{0} I a}{4 \pi} \int_{0}^{2 \pi} \frac{r \cos \theta \cos \varphi}{\left[r^{2}+a^{2}-2 a r \sin \theta(\sin \beta \sin \varphi+\cos \beta \cos \varphi)\right]^{\frac{3}{2}}} d \varphi \\
& B_{y}=\frac{\mu_{0} I a}{4 \pi} \int_{0}^{2 \pi} \frac{r \cos \theta \sin \varphi}{\left[r^{2}+a^{2}-2 a r \sin \theta(\sin \beta \sin \varphi+\cos \beta \cos \varphi)\right]^{\frac{3}{2}}} d \varphi \\
& B_{z}=\frac{\mu_{0} I a}{4 \pi} \int_{0}^{2 \pi} \frac{a-r \sin \theta(\sin \varphi \sin \beta+\cos \varphi \cos \beta)}{\left[r^{2}+a^{2}-2 a r \sin \theta(\sin \beta \sin \varphi+\cos \beta \cos \varphi)\right]^{\frac{3}{2}}} d \varphi
\end{aligned}
$$

Thus, we can get the relationship between $B$ and $I$ based on the Eq. (5). For any sensor position at point $P$, 


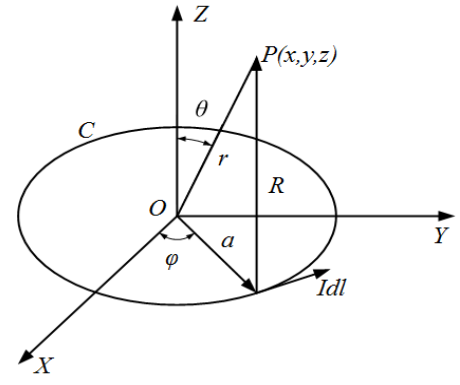

Fig. 4. Circular coil

when three groups solenoids are into the DC current asynchronously, the magnetic flux density is expressed by Eq. (6). In the Eq. (6), $B_{p m}(m=x, y, z)$ is magnetic flux density when $m$ axis solenoids are into the known DC current $I_{m} . K_{s m}$ represents the solenoid coefficient between $B_{p m}$ and $I_{m}$

$$
\left(\begin{array}{l}
B_{p x} \\
B_{p y} \\
B_{p z}
\end{array}\right)=\left(\begin{array}{ccc}
K_{s x} & 0 & 0 \\
0 & K_{s y} & 0 \\
0 & 0 & K_{s z}
\end{array}\right)\left(\begin{array}{l}
I_{x} \\
I_{y} \\
I_{z}
\end{array}\right)
$$

By Eq. (6), $B$ can be measured by a Gauss meter, and the solenoid $I$ is given by previously. So, the solenoid coefficient $K_{s}$ can be calibrated.

\section{B. The Calibration Principle of 3D Sensor Coefficient}

Single 3D MFMS consists of three square multi-turn coils seen in Fig. 2. Before experiment, check the position of the sensor and make the sensor coordinate system consist with the calibration-platform. Use $\mathrm{X}$ axis coil as an example for theoretical analysis.

The cosine form of the current

$$
i(t)=\hat{I} \cos (\omega t)
$$

is passed through $\mathrm{X}$ axis solenoids of $3 \mathrm{D}$ solenoid calibration-platform, the value of magnetic flux density and induction EMF at sensor point $P$ are

$$
B_{p}(t)=K_{s} i(t)=K_{s} \hat{I} \cos (\omega t)=\hat{B}_{p} \cos (\omega t)
$$

and

$e_{p}(t)=-\frac{d \lambda(t)}{d t}=-N \frac{d \Phi(t)}{d t}=-N S \frac{d B(t)}{d t}=N S \omega \hat{B}_{p} \sin (\omega t)$

Where $K_{s}$ is calibrated solenoid coefficient according to Eq. (6). In Eq. (9), $N$ and $S$ are the number of turns and cross-sectional area of $\mathrm{X}$ axis coil, respectively. For a particular coil on a particular sensor, $N$ and $S$ are fixed values. Make the sensor coefficient $K_{c}=N S$, and equation (9) becomes

$$
e_{p}(t)=j K_{c} \omega B_{p}(t)
$$

where $j=\frac{\pi}{2}$ replaces phase difference between $e_{p}(t)$ and $B_{p}(t)$.

For any sensor position at point $P$, when three groups solenoids are into the cosine form of the current asynchronously, the induction EMF of the three coils are

$$
\left(\begin{array}{l}
e_{p x} \\
e_{p y} \\
e_{p z}
\end{array}\right)=\omega\left(\begin{array}{ccc}
K_{c x} & 0 & 0 \\
0 & K_{c y} & 0 \\
0 & 0 & K_{c z}
\end{array}\right)\left(\begin{array}{l}
B_{p x} \\
B_{p y} \\
B_{p z}
\end{array}\right)
$$

where $e_{p m}$ is induction EMF of $m$ axis coil at point $P$, and $K_{c m}$ is the coil coefficient of $m$ axis. The value of $e_{p m}$ is measured by data acquisition card and the $B_{p m}$ can be calculated by Eq. (6). Then, $K_{c m}$ is calibrated according to Eq. (11).

In the TMS experiment, if the $K_{c}$ has been obtained by above-mentioned experimental method and $e_{p m}(t)$ waveform data has been measured, the $B_{p m}(t)$ waveform can be calculated by

$$
B_{p m}(t)=\int_{0}^{t} \frac{e_{p m}(t)}{K_{c m}} d t
$$

When $B_{p x}(t), B_{p y}(t)$ and $B_{p z}(t)$ have been calculated, we can obtained the sum of the transient magnetic flux density

$$
B_{p}(t)=\sqrt{B_{p x}^{2}(t)+B_{p y}^{2}(t)+B_{p z}^{2}(t)}
$$

\section{EXPERIMENT TESTING}

\section{A. The Experiment of 3D Solenoid Calibration- platform Calibration}

The experimental equipments for the 3D solenoid coefficients calibration include 3D solenoid calibrationplatform, signal generator, power amplifier, 3D Gauss meter and digital multimeter. The 3D Gauss meter is Model 931 digital high precision instrument which accuracy is $0.01 \%$ and resolution is $0.00001 \mathrm{mT}$. The digital multimeter is DM3068 that is a $6^{1 / 2}$ bit double display digital multimeter and the DC current measuring range from $-10.5 \mathrm{~A}$ to $10.5 \mathrm{~A}$. The whole threedimensional magnetic field calibration system is shown in Fig. 5.

The experimental steps are as follows:

Step one, fix the four-layer support at the center of the 3D solenoid calibration-platform.

Step two, put the probe of 3D Gauss meter at the sensor position $\mathrm{P} 1(x=10 \mathrm{~mm}, y=40 \mathrm{~mm}, z=-4.44 \mathrm{~mm}$ in global coordinate) and check the local coordinate of Gauss meter is consistent with the global coordinate of the 3D solenoid calibration-platform.

Step three, pass the known DC current through the X axis solenoids of the calibration-platform. The DC current 


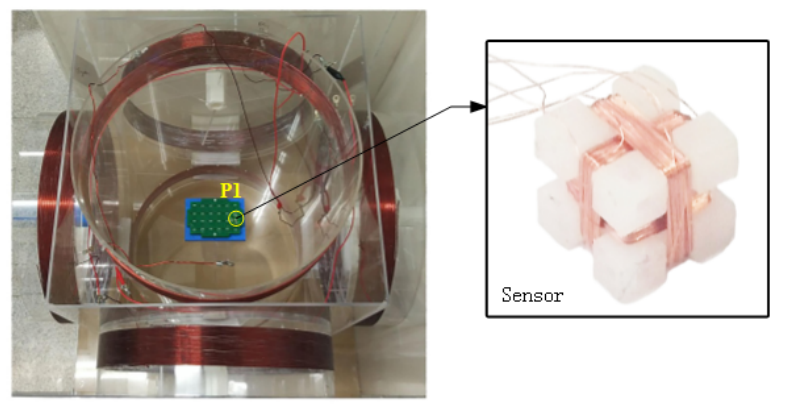

Fig. 5. Three dimensional magnetic field calibration system

range is $0-6 \mathrm{~A}$ and the increasing step is $0.5 \mathrm{~A}$ each time. Record the readings of mulitmeter $I_{x}$ and the Gauss meter $B_{x}$. According to Eq. (6), the coefficient $K_{s x}$ of the solenoid magnetic field and current at the sensor point P1 can be calibrated.

Step four, pass DC current through the $\mathrm{Y}$ and $\mathrm{Z}$ axis solenoids. Repeat step three, the coefficient $K_{s y}$ and $K_{s z}$ are also calibrated.

Final step, put the probe of Gauss meter at others 3D array sensor positions. Repeat step two to four, the solenoid coefficients at the entire 3D array sensor points are calibrated.

Based on the above experimental steps, the recording values of magnetic field and current at the sensor point P1 are shown in Table II. And the calibrated solenoid coefficient $K_{s}$ at point $P 1$ is shown in Fig. 6.

In the point $\mathrm{P} 1$, the theoretical coefficient matrix $\left[\mathrm{K}_{\mathrm{s}}\right]$ and calibrated coefficient matrix $\left[\mathrm{K}_{\mathrm{s}}{ }^{*}\right]$ of the $3 \mathrm{D}$ solenoid are

$$
K_{s}=\left[\begin{array}{ccc}
3.9278 * 10^{-5} & 0 & 0 \\
0 & 4.0900 * 10^{-5} & 0 \\
0 & 0 & 3.9187 * 10^{-5}
\end{array}\right]
$$

and

$$
K_{s}^{*}=\left[\begin{array}{ccc}
3.8952 * 10^{-5} & 0 & 0 \\
0 & 4.0639 * 10^{-5} & 0 \\
0 & 0 & 3.9544 * 10^{-5}
\end{array}\right]
$$

The theoretical coefficient matrix $\left[\mathrm{K}_{\mathrm{s}}\right]$ is calculated by Biolt-Savart law.

Based on Eq. (14), the relative error of the 3D solenoid coefficient between theoretical and calibrated value can be calculated.

$$
e_{r}\left(K_{s}^{*}\right)=\frac{\left|K_{s m}-K_{s m}^{*}\right|}{K_{s m}}, \quad(m=x, y, z)
$$

The relative error matrix is
TABLE II

Magnetic Field And CURRENT VALUe at SENSOR Point P1

\begin{tabular}{cccccc}
\hline \hline $\operatorname{Ix}(\mathrm{A})$ & $\begin{array}{c}\mathrm{Bx} \\
\text { (Gauss) }\end{array}$ & $\mathrm{Iy}(\mathrm{A})$ & $\begin{array}{c}\text { By } \\
\text { (Gauss) }\end{array}$ & $\mathrm{Iz}(\mathrm{A})$ & $\begin{array}{c}\mathrm{Bz} \\
\text { (Gauss) }\end{array}$ \\
\hline 0.4501 & 0.230 & 0.4441 & 0.194 & 0.4832 & 0.174 \\
0.9744 & 0.422 & 0.9462 & 0.414 & 1.0781 & 0.400 \\
1.4522 & 0.578 & 1.443 & 0.595 & 1.5218 & 0.603 \\
1.9553 & 0.735 & 2.0007 & 0.807 & 1.9852 & 0.795 \\
2.4511 & 0.908 & 2.4627 & 0.971 & 2.4821 & 1.012 \\
2.9798 & 1.073 & 3.0131 & 1.229 & 3.0155 & 1.227 \\
3.4540 & 1.299 & 3.4871 & 1.393 & 3.5563 & 1.442 \\
3.9606 & 1.449 & 3.9755 & 1.584 & 4.0437 & 1.595 \\
4.4483 & 1.601 & 4.4824 & 1.760 & 4.5240 & 1.832 \\
5.0127 & 1.842 & 4.9546 & 1.964 & 4.9812 & 2.019 \\
5.4952 & 2.016 & 5.4517 & 2.132 & 5.5057 & 2.187 \\
\hline \hline
\end{tabular}
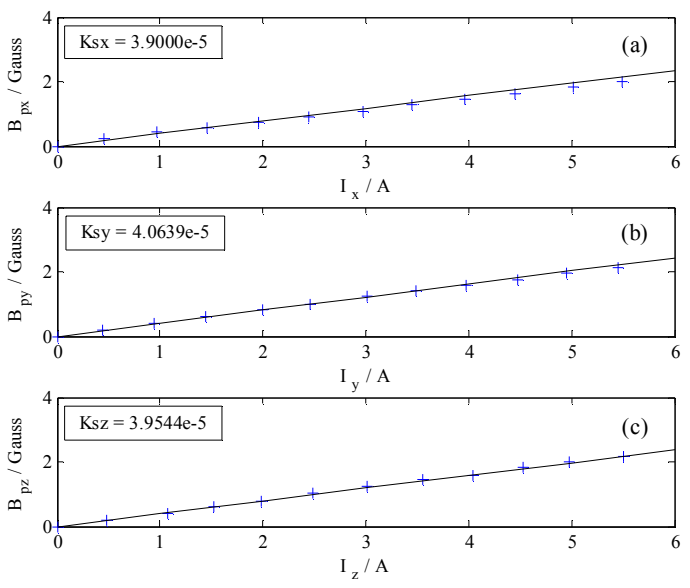

Fig. 6. Solenoid Coefficient calibration at point $\mathrm{P} 1$ (a) $\mathrm{X}$ axis, (b) $\mathrm{Y}$ axis (c) and $\mathrm{Z}$ axis

$$
e_{r}\left(K_{s}^{*}\right)=\left[\begin{array}{ccc}
0.83 \% & 0 & 0 \\
0 & 0.86 \% & 0 \\
0 & 0 & 0.91 \%
\end{array}\right] \text {. }
$$

The error is mainly caused by follow three aspects. One is winding error because the $3 \mathrm{D}$ solenoid is not an absolute tightly winding. Two is fixed error. The relative position of the $\mathrm{X}, \mathrm{Y}$ and $\mathrm{Z}$ axis solenoids exist difference with the position relationship of shown in Fig. 3. Three is instrumental measurement error and other artificial errors.

Each element of the relative error matrix $e_{r}\left(K_{s}^{*}\right)$ is less than $1 \%$ which is permitted range. Calibrate solenoid coefficients at the other $3 \mathrm{D}$ sensor points, the same error conclusion can be drawn.

The results of $e_{r}\left(K_{s}^{*}\right)$ indicate that the calibrated solenoid coefficient matrix $\left[\mathrm{K}_{\mathrm{s}}{ }^{*}\right]$ can be used for the later calibration of the $3 \mathrm{D}$ sensor coils coefficients matrix $\left[\mathrm{K}_{\mathrm{c}}\right]$.

\section{B. The Experiment of 3D Sensor Calibration}

The experimental equipments for the 3D array sensor coefficients calibration include 3D solenoid calibrationplatform, the simplified brain model, signal generator, power amplifier, digital multimeter and data acquisition card.

The experimental steps are as follows:

Step one, fix the simplified brain model at the center of 
the 3D solenoid calibration-platform.

Step two, make the data acquisition card connect to both ends of the $\mathrm{X}$ axis coil for getting inducted EMF purpose.

Step three, pass the known frequency and amplitude cosine current $i_{x}(t)$ through the $\mathrm{X}$ axis solenoids. Record the RMS readings of mulitmeter $I_{x}$ and the acquisition card $E_{x}$. According to Eq. (6) and the calibrated coefficient $K_{s x}$ beforehand, $B_{x}$ can be calculated. Based on Eq. (11), the $X$ axis coil coefficient $K_{c x}$ can be calibrated.

Step four, pass $i_{y}(t)$ an $i_{z}(t)$ through the $\mathrm{Y}$ and $\mathrm{Z}$ axis solenoids. Make the acquisition card connect with the corresponding axis coil. Repeat step three, the coefficient $K_{c y}$ of $K_{c z}$ are also calibrated.

Final step, let the acquisition card connect with the others 3D array sensor coils. Repeat step two to four, the coil coefficients of the entire 3D array sensor are calibrated.

Based on the above experimental steps, the RMS recording values of solenoid current and coil inducted EMF at the sensor point P1 are shown in Table III. The calibration coils coefficient $K_{s}$ is shown in Fig. 7 .

From the Fig. 7, the conclusion can be drawn that the inducted EMF of P1 sensor coils is linear relationship with magnetic flux density.

The sensor at $\mathrm{P} 1$ point as shown in the right side of Fig. 5, the parameter $K_{c}=N^{*}$ length* width can be calculated. The parameter of $X$ axis coil are $54 \times 3.46 \mathrm{~mm} \times 3.46$ $\mathrm{mm}, \mathrm{Y}$ axis coil are $56 \times 3.86 \mathrm{~mm} \times 3.86 \mathrm{~mm}$ and $\mathrm{Z}$ axis coil are $54 \times 4.15 \mathrm{~mm} \times 4.17 \mathrm{~mm}$. So, the theoretical coefficient matrix $\left[\mathrm{K}_{\mathrm{c}}\right]$ and calibrated coefficient matrix $\left[\mathrm{K}_{\mathrm{c}}{ }^{*}\right]$ (in Fig. 7) of the 3D sensor coils are

$$
K_{c}=\left[\begin{array}{ccc}
6.6466 * 10^{-4} & 0 & 0 \\
0 & 8.3400 * 10^{-4} & 0 \\
0 & 0 & 8.9989 * 10^{-4}
\end{array}\right]
$$

and

$$
K_{c}^{*}=\left[\begin{array}{ccc}
6.4420 * 10^{-4} & 0 & 0 \\
0 & 8.2987 * 10^{-4} & 0 \\
0 & 0 & 8.9138 * 10^{-4}
\end{array}\right]
$$

The relative error of the 3D sensor coil coefficients between theoretical and calibrated value can be calculated similarly 3D solenoid coefficients. The relative error matrix is

$$
e_{r}\left(K_{c}^{*}\right)=\left[\begin{array}{ccc}
0.37 \% & 0 & 0 \\
0 & 0.16 \% & 0 \\
0 & 0 & 0.95 \%
\end{array}\right]
$$

The error is mainly caused by follow three aspects. One is winding error because the 3D sensor coils are not an absolute tightly winding. Two is instrumental measurement error and other artificial errors. Three is
TABLE III

THE RMS VALUE OF SOLENOID CURRENT AND P1 SENSOR COILS EMF

\begin{tabular}{cccccc}
\hline \hline $\mathrm{I}(\mathrm{A})$ & $\mathrm{E}(\mathrm{mV})$ & $\mathrm{I}(\mathrm{A})$ & $\mathrm{E}(\mathrm{mV})$ & $\mathrm{I}(\mathrm{A})$ & $\mathrm{E}(\mathrm{mV})$ \\
\hline 0.492 & 0.0170 & 0.502 & 0.0233 & 0.507 & 0.0240 \\
0.997 & 0.0344 & 1.004 & 0.0466 & 1.002 & 0.0474 \\
1.487 & 0.0514 & 1.500 & 0.0699 & 1.502 & 0.0713 \\
1.992 & 0.0688 & 2.002 & 0.0932 & 2.01 & 0.0953 \\
2.497 & 0.0864 & 2.505 & 0.1166 & 2.506 & 0.1188 \\
2.989 & 0.1035 & 2.994 & 0.1394 & 3.003 & 0.1424 \\
3.489 & 0.1209 & 3.506 & 0.1632 & 3.504 & 0.1665 \\
4.021 & 0.1395 & 4.021 & 0.1872 & 4.004 & 0.1903 \\
4.499 & 0.1564 & 4.497 & 0.2094 & 4.500 & 0.2139 \\
5.004 & 0.1740 & 5.000 & 0.2328 & 5.001 & 0.2378 \\
5.495 & 0.1913 & 5.502 & 0.2565 & 5.501 & 0.2617 \\
6.000 & 0.2090 & 6.003 & 0.2799 & 6.002 & 0.2855 \\
6.490 & 0.2262 & 6.503 & 0.3031 & 6.499 & 0.3092 \\
\hline \hline
\end{tabular}
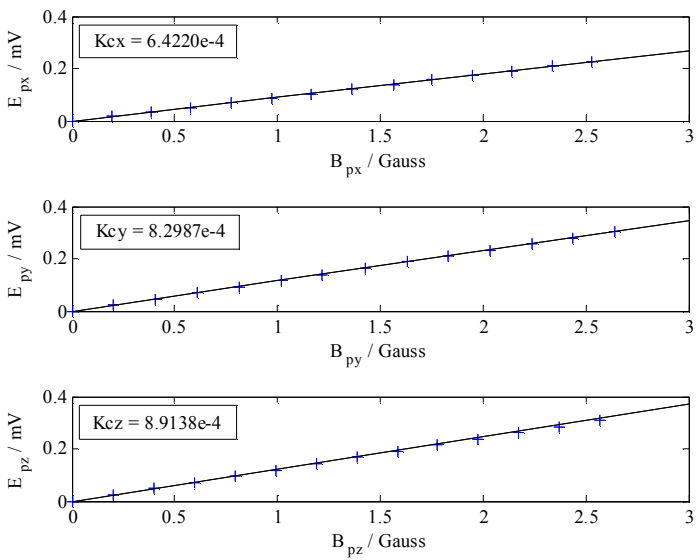

Fig. 7. P1 sensor coils coefficient calibration (a) X axis, (b) $\mathrm{Y}$ axis and (c) $\mathrm{Z}$ axis

accumulated error. The solenoid calibration coefficients are used in later magnetic flux density calculations according to Eq. (11) and Eq. (6).

Each element of the relative error matrix $e_{r}\left(K_{c}^{*}\right)$ is less than $1 \%$ which is permitted range. Calibrate coil coefficients of the other 3D sensors, the same error conclusion can be drawn. The results of $e_{r}\left(K_{c}^{*}\right)$ indicate that the calibrated 3D sensor coil coefficient matrix $\left[K_{c}^{*}\right]$ can be used for the later TMS pre-testing experiment.

Finally, the $88 \times 3$ coil coefficients $K_{C}$ of the 3D array sensor have been calibrated, so the magnetic field strength $B$ of any sensor point can be calculated by Eq. (12) and Eq. (13).

\section{CONCLUSION}

In this paper, a new simplified brain model has been presented and a new 3D array MFMS structure and 3D solenoid calibration-platform have been designed. Firstly, calibrated the solenoid coefficient of the calibrationplatform. And then calibrated the sensor coil coefficients of the 3D array MFMS. The errors of calibrated coefficients is within the allowable range of engineering errors. This result shows that the system satisfy the requirement of the design accuracy. 
Using the calibrated 3D array MFMS can obtain the magnetic field distribution in the simplified brain model while place a stimulated coil with pulse current over the model. By this reverse engineering, the 3D magnetic field measurement system has achieved the object of magnetic field measurement for TMS pre-testing. That reduces the risk of clinical surgery for TMS in some degree.

\section{REFERENCES:}

[1] A. T. Barker, R. Jalinous and I. L. Freeston, Non-invasive magnetic stimulation of human motor cortex vol. 1, 1985.

[2] A. T. Barker, I. L. Freeston, R. Jalinous, and J. A. Jarratt, "Motor responses to non-invasive brain stimulation in clinical practice," Electroencephalography \& Clinical Neurophysiology, vol. 61, p. S70, 1985.

[3] B. J. Roth, L. G. Cohen, M. Hallett, W. Friauf, and P. J. Basser, "A theoretical calculation of the electric field induced by magnetic stimulation of a peripheral nerve.," Muscle \& Nerve, vol. 13, pp. 734-41, 1990

[4] J. M. Lee and J. H. Lee, "Benefits of using transcranial magnetic stimulation as a tool to facilitate the chronic knee injury rehabilitation," Journal of Physical Therapy Science, vol. 29, pp. 733-736, 2017.

[5] B. M. Luber, S. Davis, E. Bernhardt, A. Neacsiu, L. Kwapil, S. H Lisanby, and T. J. Strauman, "Using neuroimaging to individualize TMS treatment for depression: Toward a new paradigm for imaging-guided intervention," Neuroimage, 2017.

[6] J. P. Lefaucheur, N. Andréobadia, A. Antal, S. S. Ayache, C. Baeken, D. H. Benninger, R. M. Cantello, M. Cincotta, C. M. De, and R. D. De, "Evidence-based guidelines on the therapeutic use of repetitive transcranial magnetic stimulation (rTMS)," Clinical Neurophysiology, vol. 125, pp. 2150-2206, 2014.

[7] F. Duecker and A. T. Sack, "Rethinking the role of sham TMS," Frontiers in Psychology, vol. 6, p. 210, 2015.
[8] S. Rossi, M. Hallett, P. M. Rossini, and A. Pascual-Leone, "Safety, ethical considerations, and application guidelines for the use of transcranial magnetic stimulation in clinical practice and research," Clinical Neurophysiology, vol. 120, pp. 2008-2039, 2009.

[9] Z. D. Deng, S. H. Lisanby and A. V. Peterchev, "Electric field depth-focality tradeoff in transcranial magnetic stimulation: Simulation comparison of 50 coil designs," Brain Stimulation, vol. 6, pp. 1-13, 2013.

[10] A. Laudani, F. R. Fulginei and A. Salvini, "TMS Array Coils Optimization by Means of CFSO," IEEE Transactions on Magnetics, vol. 51, pp. 1-4, 2015.

[11] Y. Roth, Y. Levkovitz, G. S. Pell, M. Ankry, and A. Zangen, "Safety and characterization of a novel multi-channel TMS stimulator.," Brain Stimulation, vol. 7, pp. 194-205, 2014.

[12] A. V. Peterchev, K. D’Ostilio, J. C. Rothwell, and D. L. Murphy, "Controllable pulse parameter transcranial magnetic stimulator with enhanced circuit topology and pulse shaping.," Journal of Neural Engineering, vol. 11, p. 056023-056023, 2014.

[13] H. Zhang, H. U. Rui-Ping, M. X. Wang, J. L. Zhang, L. U. HaiFeng, and D. U. Xiao-Xia, "A method of TMS coil positioning based on functional and structural MRI," Chinese Journal of Magnetic Resonance Imaging, 2016.

[14] H. Zhang, H. U. Rui-Ping, M. X. Wang, J. L. Zhang, L. U. HaiFeng, and D. U. Xiao-Xia, "A method of TMS coil positioning based on functional and structural MRI," Chinese Journal of Magnetic Resonance Imaging, 2016.

[15] J. D. Bijsterbosch, A. T. Barker, K. H. Lee, and P. W. R. Woodruff, "Where does transcranial magnetic stimulation (TMS) stimulate? Modelling of induced field maps for some common cortical and cerebellar targets," Medical \& Biological Engineering \& Computing, vol. 50, pp. 671-681, 2012.

[16] E. Michel, D. Hernandez and S. Y. Lee, "Electrical conductivity and permittivity maps of brain tissues derived from water content based on T1 -weighted acquisition.," Magnetic Resonance in Medicine, 2016. 